\title{
Inhalt.
}

Einleitung . . . . . . . . . . . . . . . . . 1

\section{Erstes Kapitel.}

Die klimatischen Einflüsse . . . . . . . . . . . . . . . 23

A. Der Luftdruck. . . . . . . . . . . . . . . . . . . . . . . . . 24

Örtliche und zeitliche Verteilung des Luftdrucks . . . . . . . . . 25

Hygienische Bedeutung der Luftdruckschwankungen . . . . . . . 26

B. Die Luftbewegung . . . . . . . . . . . . . . . . . . . . . . . 28

Verteilung der Iuftbewegung auf der Erdoberfläche . . . . . . . 29

Hygienische Bedeutung der Lufthewegung . . . . . . . . . . . 31

C. Dis Luftfeuchtigkeit . . . . . . . . . . . . . . . . . . . . . . 33

Verteilung der Luftfeuchtigkeit auf der Erdoberfläche . . . . . . 35

Hygienische Bedeutung der Luftfeuchtigkeit. . . . . . . . . 37

1). Die Wärme . . . . . . . . . . . . . . . . . 41

Örtliche und zeitliche Schwankungen der Temperatur . . . . . . 42

Hygienischer Finfluß der beobachteten Temperaturgrade und Temperaturschwnnkungen . . . . . . . . . . . . . . . . . . . . 45

Die Wärmeregulierung des Körpers. . . . . . . . . . . . . . 45

a) Die Einwirkung hoher Temperaturen. . . . . . . . . . 50

b) Die Einwirkung niedriger Temperaturen . . . . . . . . . . 52

E. Niederschläge . . . . . . . . . . . . . . . . . . . . 55

F. Sonnenstrahlung . . . . . . . . . . . . . . . . . . . . 56

1. Die Wärmestrahlen . . . . . . . . . . . . . . 56

2. Die Helligkeitsstrahlen . . . . . . . . . . . . . . . . . . . 57

3. Die kurzwelligen Strahlen . . . . . . . . . . . . . . . . . . 58

G. Luftelektrizität . . . . . . . . . . . . . . . . . . . . . . . . . . . 59

H. Allgemeiner Charakter von Witterung und Klima . . . . . . . . . 60

1. Die Witterung . . . . . . . . . . . . . . . . . 60

Jahreszeitliche Verteilung der Todesfälle. . . . . . . . 61

2. Das Klima . . . . . . . . . . . . . . . . . . . . . . . . . 64

a) Die tropische und (subtropische) Zone . . . . . . . . . . . 65

Krankheiten der Tropenzone. . . . . . . . . . . . . . . . 66

b) Die arktische Zone . . . . . . . . . . . . . . . . . . . . . . 67

Krankheiten des polaren Klimas . . . . . . . . . . . . . 67

c) Die gemäßigte Zone. . . . . . . . . . . . . . . . . . . . . . 68

Krankheiten der gemäßigten Zone . . . . . . . . . . . . . . . . 69

d) Das Höhenklima . . . . . . . . . . . . . . . . . . . . . . . . 70

Krankheiten des Höhenklimas . . . . . . . . . . . . . . 71

Akklimatisation . . . . . . . . . . . . . . . . . . 73 


\section{Zweites Kapitel.}

Seite

Die gas- und staubförmigen Bestandteile der Luft . . . . . . 76

I. Chemisches Verhalten . . . . . . . . . . . . . 77

1. Der Sauerstoff . . . . . . . . . . . . . . . . 78

2. Ozon und Wasserstoffsuperoxyd . . . . . . . . . . . . . . . 78

3. Kohlensäure . . . . . . . . . . . . . . . . . . . . 81

4. Sonstige gasförmige Bestandteile der Luft. . . . . . . . . . . 82

II. Der Luftstaub . . . . . . . . . . . . . . . . . . . . . . . . 91 9

1. Grob sichtbarer Staul . . . . . . . . . . . . . 92

2. Rauch und $\mathrm{Ru} B$. . . . . . . . . . . . . . . . . . 94

3. Die Sonnenstäubchen . . . . . . . . . . . . . . . . . 95

4. Die Mikroorganismen . . . . . . . . . . . . . . . . 95

\section{Drittes Kapitel.}

Der Boden . . . . . . . . . . . . . . . . . . 101

I. Oberflächengestaltung und geognostisches Verhalten . . . . . . 101

II. Die mechanische Struktur der oberen Bodenschichten. . . . . . . 102

a) Korngröße, Porenvolum, Porengröße . . . . . . . . . . . 103

b) Flächenwirkungen des Bodens . . . . . . . . . . . . . 105

III. Temperatur des Bodens . . . . . . . . . . . . . . . . . . 108

IV. Chemisches Verhalten des Bodens. . . . . . . . . . . . . . . . 110

V. Die Bodenluft. . . . . . . . . . . . . . . . . . . . . . . . . 111

VI. Verhalten des Wassers im Boden . . . . . . . . . . . . . . . 113

A. Das Grundwasser . . . . . . . . . . . . . . . . . . . . . 113

B. Das Wasser der obcren Bodenschichten. . . . . . . . . . 118

VII. Die Mikroorganismen des Bodens . . . . . . . . . . . . 122

\section{Viertes Kapitel.}

Das Wasser. . . . . . . . . . . . . . . . . . . 126

A. Allgemeine Beschaffenheit der natürlichen Wässer . . . . . . . 126

B. Die hygienischen Anforderungen an Trink- und Brauchwasser . . . 131

C. Untersuchung und Beurteilung des Trinkwassers . . . . . . . . 138

D. Die Wasserversorgung . . . . . . . . . . . . . . . 150

1. Lokale Wasserversorgung . . . . . . . . . . . . . 150

2. Zentrale Wasserversorgung . . . . . . . . . . . . 153

Eis. Künstliches Selterwasser. . . . . . . . . . . . . . . . . 167

\section{Fünftes Kapitel.}

Ernährung und Nahrungsmittel . . . . . . . . . . . . . 168

A. Die Deckung des Nährstoffbedarfs des Menschen . . . . . . . . 168

I. Die Bedeutung der einzelnen Nährstoffe . . . . . . . . . . . 168

1. Die Eiweißstoffe . . . . . . . . . . . . . . . . . 170

2. Die Fette . . . . . . . . . . . . . . . . 172

3. Die Kohlehydrate . . . . . . . . . . . . . . 173

4. Das Wasser. . . . . . . . . . . . . . . . . 174

5. Die Salze. . . . . . . . . . . . . . . . . . 175

6. Die Vitamine . . . . . . . . . . . . . . . . 175

7. Die Genuß- und Reizmittel . . . . . . . . . . 176 
1I. Quantitative Verhältnisse des Kostmaßes .... Seite

I. Erhaltung des Körperlecstaunles (Erhaltungskostma 3 ) . . . 180

2. Eiweiß-(Fleisch-)Ansatz teim Jirwachsenen . . . . . . . 182

3. Fettansatz . . . . . . . . . . . . . . 183

4. Fettverlust . . . . . . . . . . . . . . . 183

5. Wachstum ................ . . 184

III. Weitere Anforderungen an eine rationelle Kost . . . . . . . . 186

1. Die Ausnut\%harkeit und Verdaulichkeit der Nahrungsmittel 187

2. Aufbewahrung und Zuliereitung der Nahrungsmittel . . . 189

3. Das Volum der Nahrung. . . . . . . . . . . . . . 191

4. Die Temperatur der Nahrung . . . . . . . . . . . . . 192

5. Vertcilung der Tageskost auf Mahlzeiten . . . . . . . . 192

6. Schutz gegen Verfälschung der Nahrungsmittel . . . . 193

IV. Auswahl und Zusammensetzung der Kost für praktische Zwecke 193

V. Sozialhygienische Gesichtspunkte bei der Beschaffung der Kost 197

B. Die einzelnen Nahrungsmittel . . . . . . . . . . . . . . . . . . 204

1. Die Kuhmilch . . . . . . . . . . . . . . . . . . . . . . 204

a) Die Zersetzungen der Milch . . . . . . . . . . . 205

b) Die Fälschungen der Milch . . . . . . . . . . . . . . . 208

c) Krankheitserreger und Gifte der Mileh . . . . . . . . . . 209

Prophylaktische Maßregeln:

1. Die Untersuchung und Kontrolle der Milch . . . . . . . 211

2. Die Ülerwachung der Milchwirtschaften . . . . . . . 215

3. Präparation der Milch vor dem Verkauf . . . . . . . 215

4. Präparation der Milch nach dem Kauf . . . . . . . . 219

2. Die Ernährung der Kinder mit Mileh und Milchsurrogaten . . 210

3. Molkereiprodukte . . . . . . . . . . . . . . . . 227

4. Fleisch . . . . . . . . . . . . . . . . . 233

Gesundheitssclıädigungen durch Nleischgenuß:

1. Tierische Parasiten des Fleisches . . . . . . . . . 235

2. Auf pflanzlichen Parasiten Keruhende Krankheiten der Schlachttiere . . . . . . . . . . . . . . . . . 239

3. Postmortale Veränderungen des Fleisches . . . . . . 241

4. Seltenere Anomalien des Fleisches . . . . . . . . . . . 242 Prophylaktische Maßregeln:

1. Vorsichtsmaßregeln kei der Vichhaltung ... . . . 243

2. Fleischbeschau . . . . . . . . . . . . . . 245

3. Aufbewahrung des Fleisches nach dem Schlachten . . . . 248

4. Zubereitung des Fleisches . . . . . . . . . . . 250

5. Vegetabjlische Nahrungsmittel . . . . . . . . . . . 255

a) Getreide, Mehl, Brot . . . . . . . . . . . . 255

b) Reis und Mais . . . . . . . . . . . . . . . . . . . . . 261

c) Leguminosen . . . . . . . . . . . . . . . . . . . . . . . 262

d) Kartoffeln . . . . . . . . . . . . . . . . . . . . . . . . . . . . 262

e) Die übrigen Gemüse . . . . . . . . . . . . . . . . . . . 263

6. Genußmittel . . . . . . . . . . . . . . . . . . 264

a) Alkoholische Getränke. . . . . . . . . . . . . 264

b) Kaffee, Tee, Kakao . . . . . . . . . . . . . . . . . . . 270

c) Tabak . . . . . . . . . . . . . . . . . . . . . . . . . . 271

d) Gewürze . . . . . . . . . . . . . . . . . . . . 272 
Sechstes Kapitel.

Kleidung und Hautpflege . . . . . . . . . . . . . . . . . . 273

Eigenschaften der Stoffelemente der Kleidung . . . . . . . . . . 273

Eigenschaften der zu Geweben verarbeiteten Kleiderstoffe . . . . 276

Die Beziehungen der Kleidung zu Wärmeabgabe . . . . . . . 278

Beziehungen der Kleider zur Wasserdampfabgabe des Körpers . . 281

Schutz des Körpers gegen Wärmestrahlen. . . . . . . . . . . . 282

Sonstige Anforderungen an Kleidung; Schuhwerk . . . . . . . . 282

Hautpflege; Bäder . . . . . . . . . . . . . . . 285

\section{Siebentes Kapitel.}

Die Wohnung (Wohnhaus- und Städteanlagen) . . . . . . . . . . . 287

I. Vorbereitungen für den Bau des Wohnhauses . . . . . . . . . . 287

A. Wahl und Herrichtung des Bauplatzes . . . . . . . . . . . . 287

B. Die verschiedenen Formen des Wohnhauses und ihre hygienische Bedeutung . . . . . . . . . . . . . . . . . 288

C. Die Reform des städtischen Wohnungswesens . . . . . . . 294

I. Vorläufige Abhilfemaßregeln . . . . . . . . . . . . . . 294

2. Bebauungspläne und Straßenanlagen . . . . . . . . . . 295

3. Bauordnung und Wohnungskontrolle . . . . . . . . . . 299

4. Baupläne für Kleinhäuser . . . . . . . . . . . . . . . 303

5. Die Gartenstadtbewegung . . . . . . . . . . . . . . . 309

II. Fundamentierung und Bau des Hauses . . . . . . . . . . . . . 311

III. Austrocknungsfrist; feuchte Wohnungen . . . . . . . . . . . . 317

IV. Temperatur-Regulierung der Wohnräume . . . . . . . . . . . . 323

A. Temperatur-Regulierung im Sommer . . . . . . . . . . . . . 323

B. Temperatur-Regulierung im Winter . . . . . . . . . . . . . 327

a) Lokalheizungen . . . . . . . . . . . . . . . . . 332

b) Zentralheizung . . . . . . . . . . . . . . . . . . . . . 337

V. Lüftung der Wohnräume . . . . . . . . . . . . . . . . . . 350

A. Der quantitative Ventilationsbedarf . . . . . . . . . . . . . 351

B. Die Deckung des Ventilationsbedarfs. . . . . . . . . . . . . . 352

1. Natürliche und künstliche Ventilation . . . . . . . . . . . 352

2. Systeme der künstlichen Lüftung . . . . . . . . . . . . . 354

3. Anordnung der Ventilationsöffnungen . . . . . . . . . 355

4. Motoren . . . . . . . . . . . . . . 356

C. Prüfung der Ventilationsanlagen. . . . . . . . . . . . . . . 361

D. Leistung der Ventilationsanlagen . . . . . . . . . . . . . . . . . . . . . 362

VI. Beleuchtung . . . . . . . . . . . . . . . . 366

A. Tageslicht . . . . . . . . . . . . . . . . . . 366

B. Künstliche Beleuchtung. . . . . . . . . . . . . . . . . 375

VII. Entfernung der Abfallstoffe . . . . . . . . . . . . . . . . . . 386

A. Die Beschaffung der Abfallstoffe . . . . . . . . . . . . . . . . . 387

B. Gesundheitsschädigungen durch die Abfallstoffe . . . . . . . . . . 389

C. Die einzelnen Systeme zur Entfernung der Abfallstoffe . . . . 392

1. Abfuhrsysteme . . . . . . . . . . . . . . . . . . . . . . 392

Das Grubensystem . . . . . . . . . . . . . . . . . . . 392

Das Tonnensystem . . . . . . . . . . . . . . 394

Abfuhr mit Präparation der Fäkalien . . . . . . . . . . 396 
2. Schwemmkanalisation $\quad$ Seite

3. Die Separationssysteme . . . . . . . . . . . . . . . 405

4. Beseitigung des Kanalinhalts . . . . . . . . . . . . . . 409

a) Einlauf in die Flüsse . . . . . . . . . . . . . . . . . 409

b) Beseitigung lediglich der Sink- und Schwimmstoffe . . . 411

c) Beseitigung auch der gelösten organischen Stoffe . . . 418

5. Beseitigung gewerblicher Abwässer . . . . . . . . . . . . 426

6. Untersuchung der Abwässer. . . . . . . . . . . . . . . . 428

7. Der Kehricht und die Tierkadaver . . . . . . . . . . . . 430

VIII. Leichenbestattung. . . . . . . . . . . . . . . . . . . . . 432

\section{Achtes Kapitel.}

Hygienische Fürsorge für Kinder und Kranke . . . . . . . . 438

A. Das kindliche Alter . . . . . . . . . . . . . . . . . . . . . 438

1. Die Säuglingsfürsorge . . . . . . . . . . . . . . . . . . . . 438

2. Kleinkinderfürsorge . . . . . . . . . . . . . . . . . . . . . 446

3. Die schulpfichtigen Kinder . . . . . . . . . . . . . . . . . . 447

4. Die schulentlassene Jugend . . . . . . . . . . . . . . . . . . 467

Leibesübungen für die Jugend . . . . . . . . . . . . . . . . 471

B. Fürsorge für Kranke . . . . . . . . . . . . . . . . . . . . . . 472

\section{Neuntes Kapitel.}

Beruf und Beschäftigung (Gewerbehygiene). . . . . . . . . . . 483

I. Gesundheitsschädigungen durch die allgemeinen hygienischen Verhältnisse . . . . . . . . . . . . . 485

II. Gesundheitsschädigungen durch dic Beschäftigungsweise der Arbeiter . . . . . . . . . . . . . . . . . . . . . . 488

1. Alter und Körperbeschaffenheit; Arbeitszeit . . . . . . . . 488

2. Die Arbeitsräume . . . . . . . . . . . . . . . . . . . . . 489

3. Die Muskelarbeit und die Körperhaltung . . . . . . . . . . . 489

4. Schädigung der Sinnesorgane. . . . . . . . . . . . 490

5. Gesteigerter Luftdruck . . . . . . . . . . . . . . . . . . . 491

6. Exzessive Temperaturen . . . . . . . . . . . . . . . . . . 492

7. Einatmung von Staub . . . . . . . . . . . . . . . . . 493

8. Die Einatmung giftiger Gase . . . . . . . . . . . . . . . . 497

9. Beschäftigung mit giftigem Arbeitsmaterial . . . . . . . 500

10. Gefährdung der Arbeiter durch Kontagien. . . . . . . . 506

11. Unfälle . . . . . . . . . . . . . . . . . . . . . . . . 508

III. Belästigung und Schädigung der Anwohner durch Gewerbebetriebe 511

\section{Zehntes Kapitel.}

Die parasitären Krankheiten . . . . . . . . . . . . . . . . 513

A. Allgemeine Morphologie und Biologie der Mikroorganismen. . . . . 513

a) Die Faden-(Schimmel-)pilze. . . . . . . . . . . . . . 515

b) Die Actinomyzeten . . . . . . . . . . . . . . . . . . . . . . 516

c) Die Sproßpilze . . . . . . . . . . . . . . . . . . . . . . . . . . . . . . 517

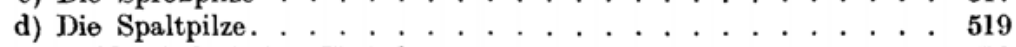

1. Morphologisches Verhaiten. . . . . . . . . . . . . . . 519

2. Lebensbedingungen . . . . . . . . . . . . . . . . . . . . 525 
Seite

Lebensaủorungen ............. . . 530

4. Absterbebedingungen . . . . . . . . . . . . . 535

5. Die diagnostische Unterscheidung und systematische Einteilung der Spaltpilzarten . . . . . . . . . . . . . 542

e) Protozoen . . . . . . . . . . . . . . . . . 544

B. Allgemeines über Verbreitungsweise und Bekämpfung der parasitären

Krankheiten . . . . . . . . . . . . . . . . . . . 547

I. Die Infektionsquellen . . . . . . . . . . . . . . . . . . . . 554

A. Beschaffenheit und Bedeutung der einzelnen Infektionsquellen 554

B. Fernhaltung, Beseitigung und Vernichtung der Infektionsquellen 556

1. Fernhaltung. . . . . . . . . . . . . . . 556

2. Mechanische Beseitigung und Abtötung der Parasiten (Desinfektion) . . . . . . . . . . . . . . 562

Mechanische Beseitigung der Keime. . . . . . . . . . . 563

Keimtötung, Desinfektion . . . . . . . . . . . 564

Formaldehyddesinfektion . . . . . . . . . . . . . . . 566

Dampfdesinfektion . . . . . . . . . . . . . . . . . . . . 569

Desinfektion von Büchern, Ledersachen usw. . . . . . . . . 573

Ausführung der Desinfektion in der Praxis . . . . . . . 575

II. Die Infektionswege . . . . . . . . . . . . . . . . . . . . 580

III. Die individuelle Disposition und Immunität . . . . . . . . . . . 585

A. Wesen und Ursachen der Disposition und Immunität . . . . . 586

I. Äußere Ursachen . . . . . . . . . . . . . . . . . . . . 586

2. Innere Ursachen. . . . . . . . . . . . . . . . . . . . . 588

a) Die Phagocytose . . . . . . . . . . . . . . . . . . . 590

b) Schutzstoffe im Blut usw. . . . . . . . . . . . . . . 592

1. Antitoxine. . . . . . . . . . . . . . . . . . . 593

2. Bakteriolysine . . . . . . . . . . . . . . 598

3. Opsonine, Bakteriotropine. . . . . . . . . . . 605

4. Agglutinine . . . . . . . . . . . . . . . . . . 607

5. Präzipitine. . . . . . . . . . . . . . . . . . . . . 610

6. Komplementbindende Antikörper (Reagine) . . . . . . 612

7. Überempfindlichkeit erzeugende Antikörper . . . . . . 614

B. Die absichtliche Herstellung der Immunität und die Schutzimpfungen ............... 619

1. Erhöhung der allgemeinen Resistenz . . . . . . . . . 6]9

2. Spezifische Schutzimpfungen . . . . . . . . . . . 620

IV. Die örtliche und zeitliche Disposition zu Infektionskrankheiten . . 627

Verbreitungsweise und Bekämpfung der einzclnen parasitären Krank-

heiten . . . . . . . . . . . . . . . . . . . . . 633

A. Schimmelpilze als Erreger von Mykosen . . . . . . . . . . . 633

B. Aktinomyzeten als Parasiten . . . . . . . . . . . . . . . . . . 635

C. Sproßpilze als Parasiten . . . . . . . . . . . . . . . . 637

D. Spaltpilze als Parasiten . . . . . . . . . . . . . . . . . . 638

1. Staphylococcus pyogenes . . . . . . . . . . . . . . . . . . 640

2. Streptococcus pathogenes . . . . . . . . . . . . . . . 643

3. Diplococcus lanceolatus . . . . . . . . . . . . . . . . . . . 646

4. Micrococcus Gonorrhoene . . . . . . . . . . . . . 649

5. Meningococcus . . . . . . . . . . . . 650 
6. Micrococcus eatarrhalis 10 Seite

7. Micr. tetragenus . . . . . . . . . . . . . . . . . 654

8. Micr. Melitensis . . . . . . . . . . . . . . . . . . . . . . 654

9. Bacillus anthracis . . . . . . . . . . . . . . . . . . . . 655

10. Bac. typhi . . . . . . . . . . . . . . . . . . . . . . 659

11. Bac. aërogenes und coli . . . . . . . . . . . . . . . . 670

12. Bac. paratyphi und enteritidis . . . . . . . . . . . . 671

13. Dysenterie- und Pseudodysenteriebazillen . . . . . . . . . . . 673

14. Baz. der hämorrhag. Sepsis . . . . . . . . . . . . . . . . . 674

15. Bac. pestis . . . . . . . . . . . . . . . . . . . . . . . 675

16. Bac. malleĩ. . . . . . . . . . . . . . . . . . . . . . . . . 681

17. Bac diphtheriae . . . . . . . . . . . . . . . . . . . . . . 683

18. Bac. tuberculosis . . . . . . . . . . . . . . . . . . . . . . 689

19. Bac. leprae. . . . . . . . . . . . . . . . . . . . . . . . 710

20. Bıc. tetani . . . . . . . . . . . . . . . . . . . 711

21. Baz. des malignen Ödems. . . . . . . . . . . . . . . . . . 713

22. Baz. des Rauschbrands . . . . . . . . . . . . . . . . . . 714

23. Bac. botulinus . . . . . . . . . . . . . . . . . . . . . . . 714

24. Bac. influenzac . . . . . . . . . . . . . . . . . 714

25. Baz. des Keuchhustens . . . . . . . . . . . . . . . . 717

26. Bac. pyocyaneus . . . . . . . . . . . . . . . . . . . 717

27. Baz. des Schweinerotlaufs. . . . . . . . . . . . . . . . 717

28. Vibrio cholerae asiaticae . . . . . . . . . . . . . . . . . . 718

E. Protozoenkrankheiten . . . . . . . . . . . . . . . . . . . . . . 729

1. Amöbendysenterie . . . . . . . . . . . . . . . . . . 730

2. Spirochaete der Febris recurrens . . . . . . . . . . . . . 731

3. Spirochaete pallida . . . . . . . . . . . . . 733

4. Trypanosen . . . . . . . . . . . . . . . . . . . . . 734

5. Piroplasmosen . . . . . . . . . . . . . . . . . . . . . . 738

6. Malaria . . . . . . . . . . . . . . . . . . . . . . . 740

F. Parasitäre Erkrankungen mit unsichtbaren, Bakterienfilter passierenden

Erregern . . . . . . . . . . . . . . 751

1. Variola . . . . . . . . . . . . . . . . . . . . . 751

2. Scharlach . . . . . . . . . . . . . . . . . . . . . . . 765

3. Masern . . . . . . . . . . . . . . . . . . . . . . . . . . 766

4. Flecktyphus . . . . . . . . . . . . . . . . . . . . . . . 767

5. Poliomyelitis, Kinderlähme . . . . . . . . . . . . . . . . . 769

6. Körnerkrankheit . . . . . . . . . . . . . . . . . . . . . . 769

7. Gelbfieber . . . . . . . . . . . . . . . . . . . . . 771

8. Danguefieber, Pappatacifieber . . . . . . . . . . . . . 771

9. Hundswut, Lyssa. . . . . . . . . . . . . . . . 772

\section{Anhang.}

Die wichtigsten Untersuchungsmethoden . . . . . . . . . . 776

I. Allgemeine Methodik der bakteriologischen Untersuchung . . . . 776
A. Mikroskopische Untersuchung . . . . . . . . . . . . 776

B. Kulturverfahren . . . . . . . . . . . . . . . . . . . . . . 782

C. Serumdiagnostik . . . . . . . . . . . . . . . . 784 
II. Spezielle parasitologische Diagnostik Seite

1. Abdominaltyphus . 792

2. Bazilläro Dysenterie . . . . . . . . . . . . . . . . . . 796

3. Cholera . . . . . . . . . . . . . . . . . . . . . . . . 797

4. Pest. . . . . . . . . . . . . . . . . . . . . . . . 802

5. Genickstarre . . . . . . . . . . . . . . . . . . . . 806

6. Diphtherie . . . . . . . . . . . . . . . . . . . 807

7. Tuberkulose . . . . . . . . . . . . . . . . . . . . 810

8. Syphilis . . . . . . . . . . . . . . . . . . . . . . . 811

9. Gonorrhoe . . . . . . . . . . . . . . . . . . . . . . . 812

10. Malaria, Trypanosen usw. . . . . . . . . . . . . . . . . 812

11. Tollwut . . . . . . . . . . . . . . . . . . . . 814

III. Physikalisch-chemische Untersuchungsmethoden . . . . . . . . . 815

1. Bestimmung der Luftfeuchtigkeit . . . . . . . . . . . . 815

2. Bestimmung des $\mathrm{CO}_{2}$-Gehaltes der Luft . . . . . . . . . 817

3. Untersuchung der Luft auf Kohlenoxyd und schweflige Säure $\mathbf{8 2 0}$

4. Chemische Trinkwasseranalyse . . . . . . . . . . . . . . 821

A. Organische Stoffe . . . . . . . . . . . . . . . . . 821

B. Ammoniak . . . . . . . . . . . . . . . . . . . . . 822

C. Salpetrige Säure. . . . . . . . . . . . . . . . . . . 822

D. Salpetersäure . . . . . . . . . . . . . . . . . . . 822

E. Chloride . . . . . . . . . . . . . . . . . . . . . . 823

F. Härte . . . . . . . . . . . . . . . . . . . . . . . 823

G. Eisennachweis . . . . . . . . . . . . . . . . . . . . 824

H. Mangannachweis. . . . . . . . . . . . . . . . . . . 825

I. Bestimmung der elektrischen Leitfähigkeit . . . . . . . 825

Register . . . . . . . . . . . . . . . . . . . . 827

\section{Berichtigungen.}

S. 179 ist in der Tabelle bei ,Kartoffeln" statt ,40\%" zu setzen , $25 \%$ ".

Ferner ist in der 4. Zeile unter der Tabelle statt ,dabei aber" zu setzen ,,beziehungsweise:".

S. 193 ist in der Zeile oberhalb der Tabelle zuzufügen ,(ohne Berücksichtigung der Abfälle)".

Ferner in der Tabelle, 2. Zeile, statt „Fleisch" zu setzen ,Fisch".

S. 731 Zeile 3 von unten statt ,(Wanzen, Flöhe)“ zu setzen ,(Läuse)“.

S. 734 letzte Zeile: ,neun" statt ,acht".

S. 767 Zeile 16 von unten: hinter „und“ ist einzufügen „bei einer äbnlic:hen im Felsengebirge beobachteten Krankheit durch".

S. 767 Zeile 16 von unten ist statt ,bis 4 'Tage“ zu setzen ,zwischen 5 bis 7 Tagen". 